mutagenesis is not suitable for all cell types, and can require substantial optimization for each application. Furthermore, the covalent bond that is cleaved to remove the bulky side chain from the light-sensitive tyrosine residue can be broken only by using high-energy light (wavelengths of less than 400 nanometres), which is toxic to living cells. These are likely to be short-term obstacles, however, because many laboratories are actively pursuing and improving methods for altering proteins in cultured cells, and even in vivo.

With its remarkable simplicity and generality, CAGE-prox opens the door to studies of previously inaccessible cellular pathways, and of the spatio-temporal control of processes that determine cell behaviour. The range of applications that Wang et al. have already proved in principle for their technique is remarkable. No doubt, many more will soon follow. -

Klaus Michael Hahn is in the Department of Pharmacology, University of North Carolina at Chapel Hill, Chapel Hill, North Carolina 27599, USA.

\title{
Superconductivity near room temperature
}

For a century, researchers have sought materials that superconduct - transport electricity without loss - at room temperature. Experimental data now confirm superconductivity at higher temperatures than ever before. SEE LETTER P.528

\section{JAMES J. HAMLIN}

$\mathrm{M}$ aterials known as superconductors transmit electrical energy with $100 \%$ efficiency. They have a wide range of applications, such as magnetic resonance imaging in hospitals. However, these applications have been hampered, largely because the superconducting state exists only at temperatures well below room temperature ( 295 kelvin). On page 528, Drozdov et al. ${ }^{1}$ report several key results that confirm that, when compressed to pressures of more than one million times Earth's atmospheric pressure, lanthanum hydride compounds become superconducting at $250 \mathrm{~K}$ - a higher temperature than for any other known material.

Superconductivity was first discovered ${ }^{2}$ in 1911, in mercury cooled below $4 \mathrm{~K}$. The temperature below which a material becomes superconducting is called the critical temperature. It was quickly appreciated that a state exhibiting zero electrical resistance could be tremendously useful, if materials that have critical temperatures much higher than $4 \mathrm{~K}$ could be found. Over the past century, as more superconductors have been discovered, the record for the highest critical temperature achieved has progressed, in fits and starts, towards the ultimate goal of room temperature.

In 2014, some of the authors of the current paper and their colleagues shattered the previous record ${ }^{3}$ of $164 \mathrm{~K}$. They found that hydrogen sulfide - a chemical responsible for the odour of rotten eggs - transforms into a superconductor at a temperature of about $200 \mathrm{~K}$, when compressed to nearly 2 million times atmospheric pressure $e^{4,5}$. Then, in 2018, two independent research groups reported, almost simultaneously, that compressed lanthanum hydride compounds might exhibit superconductivity at even higher temperatures, ranging from $215 \mathrm{~K}$ to perhaps as high as $280 \mathrm{~K}$ (refs 6-8).

The common features of these hydrogen sulfide and lanthanum hydride superconductors are that they are rich in hydrogen and that superconductivity emerges only under

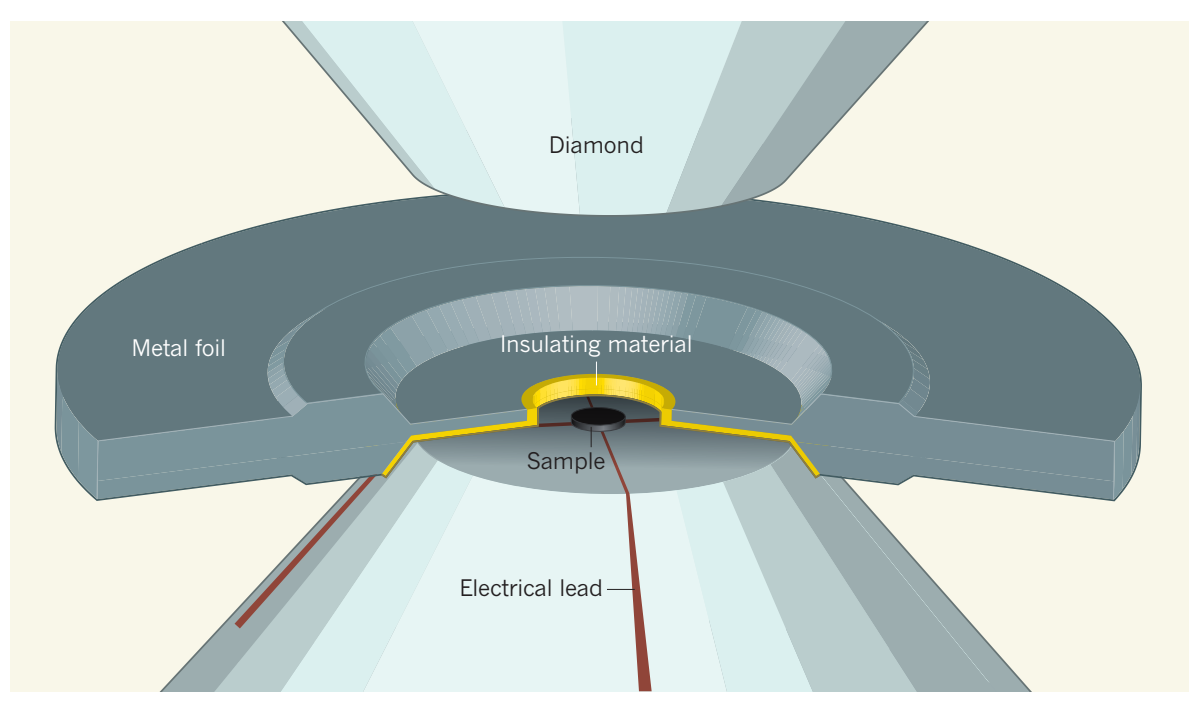

Figure 1 | Detection of high-temperature superconductivity. Drozdov et al. ${ }^{1}$ report an experiment in which a tiny sample of lanthanum is enclosed inside a hole in a thin metal foil. The hole is filled with liquid hydrogen (not shown). Four electrical leads make contact with the sample but are electrically isolated from the foil by an insulating material. The sample is squeezed between two diamonds and transforms into lanthanum hydride at high pressure. The authors use this set-up to demonstrate that lanthanum hydride becomes superconducting at a temperature of 250 kelvin, under pressures greater than one million times Earth's atmospheric pressure. ing a sample, contained by a thin metal foil, between two flattened diamonds (Fig. 1). This configuration severely limits the types of measurement that can be carried out, because the sample is tiny (of the order of 0.01 millimetres across) and is surrounded on all sides by the comparatively massive foil and diamonds. Moreover, to obtain electrical measurements, electrical leads need to make contact with the sample, but must remain electrically isolated from the foil.

e-mail:khahn@med.unc.edu

1. Rost, B. R., Schneider-Warme, F., Schmitz, D. \& Hegemann, P. Neuron 96, 572-603 (2017).

2. Dagliyan, O. \& Hahn, K. M. Curr. Opin. Struct. Biol. 57, 17-22 (2019).

3. Wang, J. et al. Nature $\mathbf{5 6 9}$, 509-513 (2019).

4. Arbely, E., Torres-Kolbus, J., Deiters, A. \& Chin, J. W. J. Am. Chem. Soc. 134, 11912-11915 (2012).

5. Krall, N., da Cruz, F. P., Boutureira, O. \& Bernardes, G. J. L. Nature Chem. 8, 103-113 (2016).

6. Chin, J. W. Annu. Rev. Biochem. 83, 379-408 (2014).

This article was published online on 8 May 2019.

pressures above about one million times atmospheric pressure. Under these extreme conditions, chemical bonds can be greatly altered, inducing the formation of compounds that are otherwise unstable. In the case of lanthanum hydride, high pressure seems to stabilize the formation of a compound, $\mathrm{LaH}_{10}$, that has a much larger hydrogen content than is achievable at ambient pressure ${ }^{9,10}$.

Drozdov et al. reached these extraordinarily high pressures (roughly half the pressure at Earth's core) using a device called a diamond anvil cell. This device could fit in the palm of your hand and generates pressure by squeez- 
The authors overcame these experimental challenges and uncovered crucial evidence that confirms the high-temperature superconductivity in lanthanum hydride compounds. To verify that a material is superconducting, researchers typically look for three features: zero electrical resistance; a reduction in the critical temperature under an applied magnetic field; and an expulsion of magnetic fields from the interior of the material on cooling (a phenomenon known as the Meissner effect). Drozdov et al. detected the first two of these signatures of superconductivity. The final criterion - observation of the Meissner effect - is currently unattainable because the samples are too small.

The search for high-temperature superconductivity in hydrogen-rich compounds can be linked to predictions ${ }^{11}$ made in 2004. The reasoning behind these predictions hinges on a theory that forecasts that, under certain circumstances, elements that have low atomic masses can contribute to high critical temperatures. Hydrogen, being the lightest element, is optimal for high critical temperatures. And, by this logic, replacing hydrogen with the heavier isotope deuterium should lower the critical temperature. Drozdov et al. observed this isotope effect and found that, compared with the lanthanum hydride samples, the critical temperature in lanthanum deuteride samples is lower by almost exactly the amount predicted by the theory.

From a scientific standpoint, these results suggest that we might be entering a transition from discovering superconductors by empirical rules, intuition or luck to being guided by concrete theoretical predictions. The superconducting critical temperature has long been considered to be one of the most difficult properties to compute accurately. But the hydrogen sulfide and lanthanum hydride experiments were motivated by computational results that anticipated both the necessary pressures and the resulting critical temperatures ${ }^{9,12}$. These remarkable successes for theory seem to be driven by innovative computational methods that were enabled by advances in computing power.

What is the practical importance of superconductivity in materials synthesized in minuscule quantities at more than one million times atmospheric pressure? The answer depends on whether the superconducting states can be recovered at ambient pressure. Diamond itself is an example of a material that forms at high pressure but is metastable at ambient pressure. The effort to manufacture synthetic diamond provided substantial motivation for the development of high-pressure methods. Today, however, synthetic diamonds are grown using a low-pressure technique called chemical-vapour deposition. Optimistically, it might eventually be possible to use similar low-pressure methods to produce metastable superconducting compounds that are initially discovered at high pressure.
In the next few years, experiments will probably focus on searching for superconductivity in other pressurized hydrogen-rich materials. Given that only a small fraction of possible hydrogen-rich systems have been subjected to experiments at these tremendous pressures, it seems more likely than ever that the dream of room-temperature superconductivity might be realized in the near future. At that point, the grand challenge will shift from pushing the necessary temperatures higher to pushing the required pressures lower.

James J. Hamlin is in the Department of Physics, College of Liberal Arts and Sciences, University of Florida, Gainesville, Florida 32611, USA. e-mail:jhamlin@ufl.edu

1. Drozdov, A. P. et al. Nature 569, 528-531 (2019).

2. Onnes, H. K. Commun. Phys. Lab. Univ. Leiden. Suppl. 29 (1911).

3. Gao, L. et al. Phys. Rev. B 50, 4260-4263 (1994).

4. Drozdov, A. P., Eremets, M. I. \& Troyan, I. A. Preprint at https://arxiv.org/abs/1412.0460 (2014).

5. Drozdov, A. P., Eremets, M. I., Troyan, I. A Ksenofontov, V. \& Shylin, S. I. Nature 525, 73-76 (2015).

6. Drozdov, A. P. et al. Preprint at https://arxiv.org/ abs/1808.07039 (2018)

7. Somayazulu, M. et al. Preprint at https://arxiv.org/ abs/1808.07695 (2018).

8. Somayazulu, M. et al. Phys. Rev. Lett. 122, 027001 (2019).

9. Liu, H., Naumov, I. I., Hoffmann, R., Ashcroft, N. W. \& Hemley, R. J. Proc. Natl Acad. Sci. USA 114 6990-6995 (2017).

10.Peng, F. et al. Phys. Rev. Lett. 119, 107001 (2017). 11.Ashcroft, N. W. Phys. Rev. Lett. 92, 187002 (2004). 12.Duan, D. et al. Sci. Rep. 4, 6968 (2014).

\section{Genome construction amends building codes}

The biggest synthetic genome so far has been made, with a smaller set of amino-acid-encoding codons than usual - raising the prospect of encoding proteins that contain unnatural amino-acid residues. SEE ARTICLE P.514

\section{BENJAMIN A. BLOUNT \& TOM ELLIS}

$\mathrm{O}$ ver the past decade, decreases in the costs of chemically synthesizing DNA and improved methods for assembling DNA fragments have enabled researchers to scale up synthetic biology to the level of generating entire chromosomes and genomes. So far, synthetic DNA has been constructed with up to one million base pairs, notably a set of chromosomes from the yeast Saccharomyces cerevisiae and several versions of the genome of the bacterium Mycoplasma mycoides ${ }^{1,2}$. Now, on page 514, Fredens et al. ${ }^{3}$ report the completion of a 4-million-base-pair synthetic version of the Escherichia coli genome. This is a landmark in the emerging field of synthetic genomics, and finally applies the technology to the laboratory's workhorse bacterium.

Synthetic genomics offers a new way of understanding the rules of life, while at the same time moving synthetic biology towards a future in which genomes can be written to design. The pioneers in the field - the researchers at the J. Craig Venter Institute in Rockville, Maryland - have used this method to better define the minimal set of genes required for a free-living cell. By adopting an approach that involves redesigning genome segments by computer, chemically synthesizing the fragments and then assembling them, these pioneers succeeded ${ }^{2}$ in reducing the size of the M. mycoides genome by around $50 \%$. Doing the same with just genome-editing tools would be much more laborious, as past work with E. coli demonstrates: here, gene-deletion methods have removed, at best, only $15 \%$ of the genome ${ }^{4}$.

Fredens and colleagues used this reduced genome from E. coli as the template for a synthetic genome with another kind of minimization in mind - codon reduction. The genetic code has inherent redundancy: there are 64 codons (triplets of 'letters', or bases) to encode just 20 amino acids plus the 'start' and 'stop' points that mark the beginning and end of a stretch of protein-coding sequence. This redundancy means, for example, that there are six codons that encode the amino acid serine, and three possible stop codons. Through design, synthesis and assembly, Fredens et al. ${ }^{3}$ have been able to construct an E. coli genome that uses only 61 of the 64 available codons in its protein-coding sequences, replacing two serine codons and one stop codon with synonyms (codons that are 'spelt' differently but give the same instruction). Past work using genome-editing tools has already produced a synthetic $E$. coli that uses just 63 of the 64 codons, but this required only the stop codons with the sequence TAG (of which there were just 321 around the genome) to be changed to an alternative stop codon ${ }^{5}$. Reduction to 61 codons demanded that a whopping 18,214 codons be changed, necessitating a genome-synthesis approach.

Fredens and colleagues built their synthetic 